\title{
Using Wave Characteristic Analysis to Study T2D Patient's Personality Traits and Psychological Behavior (GH-Method: Math-Physical Medicine)
}

\section{Gerald C Hsu}

eclaireMD Foundation, USA

\section{Introduction}

The author has contemplated a specific question:

Why do some type 2 diabetes (T2D) patients choose to face serious complications, including death, rather than change their lifestyle in order to control their diabetic conditions?

This paper provides his first attempt to understand the reasoning from a psychological viewpoint by using a quantitative wave characteristic analysis. It also links T2D patient's psychological issues and his physiological conditions together in a quantitative manner.

\section{Method}

The author collected 17,046 glucose data during a period of 241 days (5/5/2018-12/31/2018). At first, he decomposed and segregated these data into 723 postprandial plasma glucose (PPG) waveforms, and then re- integrated them into three distinctive general patterns based on wave characters, including frequency, period, wavelength, amplitude, and wave shape:

1. First pattern, Himalaya (lower frequency and very long period) has reached to a high peak and then maintaining at this plateau due to physical inactivity.

2. Second pattern, Grand Canyon (lower frequency and short period) has reached to a high peak but then decaying rapidly and stay at the trough due to "correct" amount and pattern of post-meal exercise.

3. Third pattern, Twin Peak (higher frequency and shorter period) has reached to a high crest (P1) and then dropping for a while but rising to a second but lower peak (P2) again due to insufficient post-meal exercise.

The height of glucose peak will reveal the patient's willpower on carbs/sugar intake control. However, the glucose wave pattern, particularly the waveform shape and decaying speed will disclose the patient's willpower and persistence on post-meal exercise control.

\section{Results}

The list below includes the following:

Waveform Pattern distribution \%, Peak glucose, and Average glucose.
Corresponding author

Gerald C Hsu, eclaireMD Foundation, USA

Submitted: 14 April 2020;Accepted:20 April2020;Published:01 May 2020

Himalaya: $13 \%, 157,142$

Twin Peak: 35\%, 159, 150

Grand Canyon: 52\%, 151, 137

Effective average PPG: $136 \mathrm{mg} / \mathrm{dL}$

Excessive energy from PPG: $36 \%$

Increased CVD risk \%: $1.7 \%$

(From $26.4 \%$ to $28.1 \%$ based on 2017 data, or $6 \%$ increase on CVD risk)

1. Himalaya pattern (13\% or 94 meals) includes $66 \%$ from air traveling (63 meals) and 34\% from rainy days (31 meals).

2. Twin Peak pattern (35\% or 256 meals) reveals that, initially, the patient lacked detailed knowledge on how to effectively combine both carbs/sugar and post-meal exercises (including both intensity and time span).

3. Grand Canyon pattern (52\% or 373 meals) indicates that, most of the time, he has strong willpower and discipline to control both carbs/sugar intake and post-meal exercise.

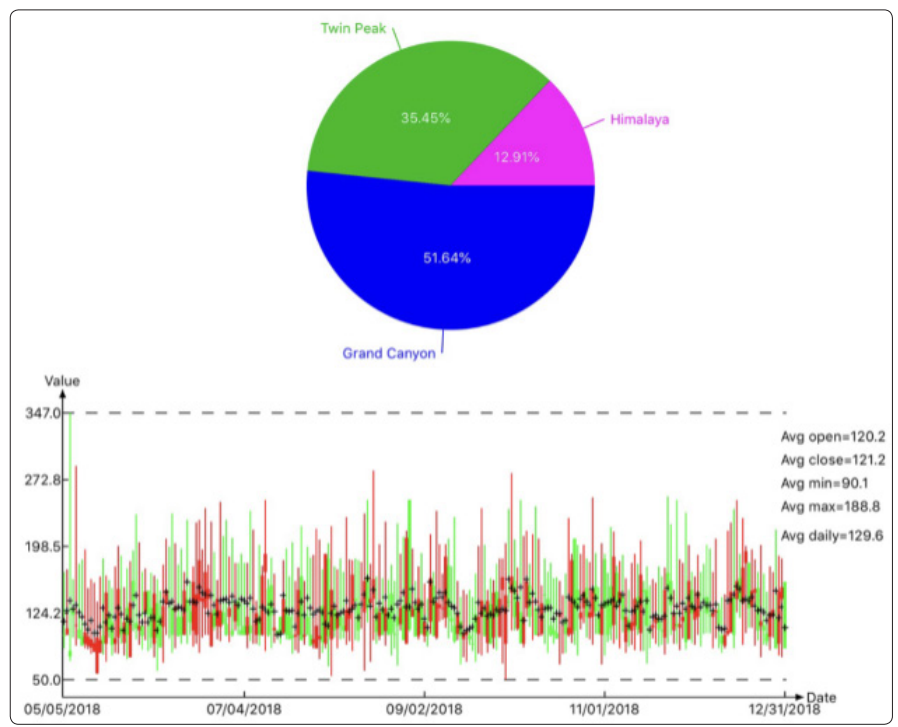

Figure 1: Pattern distribution (\%) and daily glucose candlestick chart 


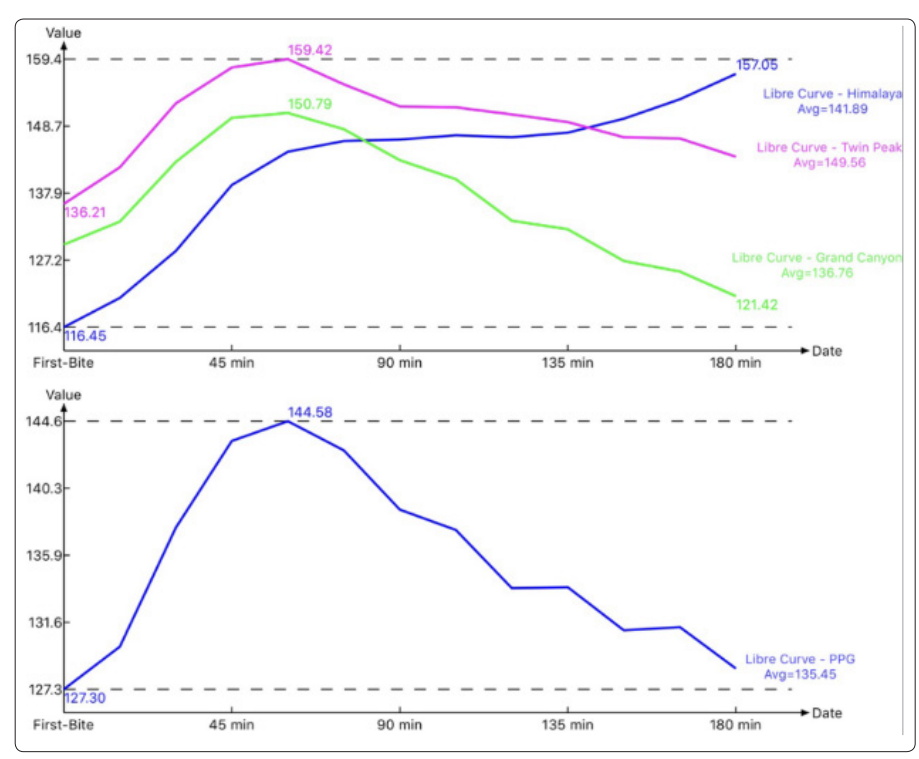

Figure 2: Three distinctive PPG waveforms shapes with average and peak glucose values $(\mathrm{mg} / \mathrm{dL})$

\section{References}

1. Hsu Gerald C (2018) Using Math-Physical Medicine to Control T2D via Metabolism Monitoring and Glucose Predictions. Journal of Endocrinology and Diabetes 1: 1-6.

2. Hsu Gerald C (2018) Using Math-Physical Medicine to Analyze Metabolism and Improve Health Conditions. Video presented at the meeting of the 3rd International Conference on Endocrinology and Metabolic Syndrome Amsterdam, Netherlands.

3. Hsu Gerald C (2018) Using Signal Processing Techniques to Predict PPG for T2D. International Journal of Diabetes \& Metabolic Disorders 3: 1-3.

4. Hsu Gerald C (2018) Using Math-Physical Medicine and Artificial Intelligence Technology to Manage Lifestyle and Control Metabolic Conditions of T2D. International Journal of Diabetes \& Its Complications 2: 1-7.
Copyright: (02020 Gerald C Hsu. This is an open-access article distributed under the terms of the Creative Commons Attribution License, which permits unrestricted use, distribution, and reproduction in any medium, provided the original author and source are credited. 